\title{
Two-Dimensional Pheromone Propagation Controller
}

\author{
Der-Shui Lee and An-Chen Lee
}

\begin{abstract}
Traditionally, the semiconductor fabrication conducts several measurements within a wafer and condenses the measurements into output quality characteristics to progress advanced process control (APC). Therefore, traditional APC employs performance indices along "time" axis and losses the space information among different measurements within a wafer. In this paper, we present a new perspective on process control with pheromones propagation mechanism, in which we treats the disturbances within a two-dimensional layout wafer as digital pheromones,. Our novel space-effect algorithm is called the two-dimensional pheromone propagation controller (2D-PPC). The simulation results show 2D-PPC, which involves space-effect, can improve the uniformity of the wafer over the conventional time-effect controllers.
\end{abstract}

Index Terms-Two-dimensional pheromone propagation controller, process control, two-dimensional pheromone basket, two-dimensional digital pheromone infrastructure, swarm intelligence

\section{INTRODUCTION}

In semiconductor manufacturing, run-to-run control adjusts the recipe slightly based on in-line measurements to even out disturbances. Disregarding the methodology of the run-to-run control, some researches condense measurements to output quality characteristics [1]-[10]. Thus, traditional run-to-run controllers like EWMA employ the "time-effect", which means disturbance of a wafer affects a measurement or the output quality characteristics at different runs, among observed data to calculate the recipe for the next run and does not consider the "space-effect", which means disturbance of a wafer affects several measurements at the same time, among measurements within a wafer at a run.

In this paper, we describe an algorithm that includes the effect among different measurements within a wafer. The concept comes from the observation that a disturbance of a wafer will affect a piece area, which may contain several measurements. The new algorithm uses swarm intelligence by assuming that the measurements have their own behavior and affect others nearby within a wafer at a run. Then, the intercepts with disturbances included of a linear regression model at different measurements within a wafer are modeled as digital pheromones. The interaction among the digital pheromones is modeled by a propagation mechanism. Because measurements within a wafer are a two-dimensional

Manuscript received August 10, 2012; revised December 11, 2012. The authors would like to thank the National Science Council of the Republic of China for financially supporting this paper under Contract NSC 100-2221-E-009-063-MY2.

D. S. Lee was with the Dept. of Mechanical Engineering, National Chiao Tung University, Taiwan (e-mail: dslee605@ ms37.hinet.net).

A. C. Lee is with the Dept. of Mechanical Engineering, National Chiao Tung University, Taiwan (e-mail: aclee@ mail.nctu.edu.tw). layout, our novel algorithm is called the two-dimensional pheromone propagation controller (2D-PPC). This study modifies the propagation-out ratio of digital pheromone infrastructure [11]-[12] to achieve 2D-PPC.

Fig. 1 is the concept of 2D-PPC. For easy interpretation and maximum coverage with minimum measurements, this study assumes that the wafer has 12 measurements in the triangular coordinate (hexagonal grids). In Fig. 1, measurements are divided into two circles, measurements 1-6 are in the outer circle and the others are in the inner circle, and the arrow means that measurements affect others nearby. In addition, after forecasting intercept of measurements independently by the time-effect (one-dimensional) controller, the two-dimensional intercept predictor, which is the realization of space effect controller, modifies the forecasting intercept of the 1D controller to forecast intercept of the next run. In other words, 2D-PPC interlaces time and space effects at a run. Then, the process recipe for the next run can be obtained by process models of every measurement.

We conducted simulations to compare the performance of PPC controllers with and without 2D-PPC. The two dimensional anthropogenic disturbance is conducted from DOE data of CMP process and the candidate controllers are examined by average and standard deviation within a wafer in the simulations. The controller parameters such as the propagation parameters of PPC and 2D-PPC were obtained from training data with minimum sum square error. The simulation results show 2D-PPC, which involves space-effect, can improve the ones of time-effect controllers.

The rest of this paper is organized as follows: the two dimensional digital pheromone infrastructure; the 2D-PPC controller structure; simulation results; conclusion and future works

\section{The Two-Dimensional Digital Pheromone INFRASTRUCTURE}

The concept of the space-effect 2D-PPC comes from the appearance that a measurement in a wafer will be affected by its nearby measurements within the same wafer in semiconductor fabrication. This section will introduce the two dimensional digital pheromone infrastructure which includes pheromone basket pheromone states, transition parameters and transition functions.

\section{A. Pheromone Basket}

The pheromone basket is the pheromone propagation environment. The "two-dimensional" PPC is named by the shape of pheromone basket being a two-dimensional plane. The environment of two dimensional digital pheromone infrastructure is a tuple $\langle B, N\rangle$, where $B$ is a finite set of 
positions $b_{m_{2}} \in B: m_{2}=1,2, \ldots, \mathrm{M}_{2}$ within the pheromone basket with size $\mathrm{M}_{2}$ and the subscript 2 means two-dimensional. Specifically, $b_{m_{2}}$ of 2D-PPC maps positions at the space and does not map the intercept of the specific run at the same measurement like PPC. Then,
$N\left(b_{m_{2}}\right) \subseteq B$ is a finite set of neighbors of $b_{m_{2}}$ and $\left|N\left(b_{m_{2}}\right)\right|$ is the size of $N\left(b_{m_{2}}\right)$. In addition, this study assumes that $b_{m_{2}}$ will accept propagation inputs from $N\left(b_{m_{2}}\right)$ without preconditions.

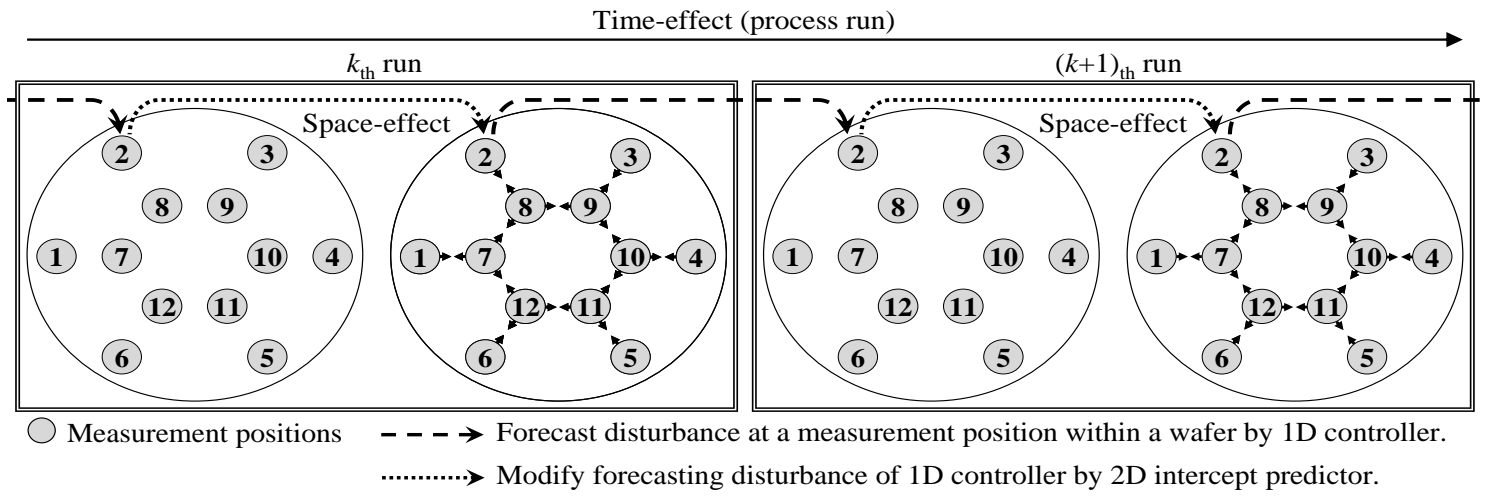

Fig. 1. Concept of space-time-effect 2D-PPC.

Then, the pheromone basket is initially empty, and then fills with $\mathrm{M}_{2}$ intercepts within the same wafer at each time stamp (or run). The external input of two dimensional pheromone basket is a finite set $R_{2}(k, i)=\left\{r_{2}\left(k, b_{m_{2}}, i\right) \in(-L, L): m_{2}=1,2, \ldots, M_{2}\right\}$, where $k=1,2, \ldots$, is the run number of the manufacturing process, $i \in \mathfrak{N}$ is the number of iterations (or propagations) in the transition functions, and $L \in \mathfrak{R}$ is the global limit of the external inputs in the environment $\langle B, N\rangle$. Since the external input is the initial condition for launching transition functions of pheromone propagation, $r_{2}\left(k, b_{m_{2}}, 0\right)$ maps to the intercepts of position $b_{m_{2}}$ at the run $k$ and $r_{2}\left(k, b_{m_{2}}, i\right)$ is 0 when $i$ is larger than 0 .

\section{A. Pheromone States}

Like [11]-[12], the states in the two dimensional pheromone basket $\langle B, N\rangle$ are $Q_{2}(k, i)$ and $S_{2}(k, i)$, where $Q_{2}(k, i)=\left\{q_{2}\left(k, b_{m_{2}}, i\right) \in \mathfrak{R}: m_{2}=1,2, \ldots, M_{2}\right\}$ is a finite set of the propagated inputs at run $k$ and iteration $i$, and $S_{2}(k, i)=\left\{s_{2}\left(k, b_{m_{2}}, i\right) \in \mathfrak{R}: m_{2}=1,2, \ldots, M_{2}\right\}$ is a finite set of the aggregated pheromones at run $k$ and iteration $i$. Therefore, $q_{2}\left(k, b_{m_{2}}, i\right)$ is regarded as the propagated input from $N\left(b_{m_{2}}\right)$ to $b_{m_{2}}$ at iteration $i$ and run $k$. Similarly, $s_{2}\left(k, b_{m_{2}}, i\right)$ is regarded as the aggregated pheromone of $b_{m_{2}}$ at iteration $i$ and run $k$. Then, $Q_{2}(k, 0)=0$ and $S_{2}(k, 0)=0$ are assumed to be the initial conditions. While launching transition functions as shown in the following sections, $Q_{2}(k, i)$ disseminates pheromones and $S_{2}(k, i)$ aggregates pheromones simultaneously.

\section{B. Pheromone Transition Parameters}

By [11]-[12], two transition parameters of the two-dimensional digital pheromone infrastructure are the evaporation parameter $E_{2} \in(0,1]$ and the propagation parameter $F_{2} \in[0,1)$. In $2 \mathrm{D}$-PPC, the propagation parameter $F_{2}$ describes the effect of a measurement on other nearby measurements within the same wafer, and the evaporation parameter $E_{2}$ indicates that the importance of the measurement data will "evaporate" with time. Because measurements within a chamber at a time stamp can be treated as a non-dissipative system, the modified pheromone infrastructure uses $E_{2}=1$ in a small pheromone basket [12].

\section{Transition Functions}

Reference [12] modified transition functions of digital pheromone infrastructure [11] to erase the boundary effect for developing PPC. This section will correct the propagation-out ratio at the frontier points of a plane pheromone basket to erase the boundary effect.

For interpreting the interactions easily and obtaining maximum coverage with minimum measurements, the authors employ triangular grids with $M_{2}=12$. The layout of the 12 measurements is shown in Fig. 1. The propagation-out ratio at the frontier points of Fig. 1 needs to modified from $F_{2} /\left|N\left(b_{m_{2}}\right)\right|[11]$ or $F_{2} /\left(2-F_{2}\right)$ [12] to $F_{2} /\left(3-2 F_{2}\right)$.

Then, the transition functions become

$$
\begin{aligned}
& s_{2}\left(k, b_{m_{2}}, i+1\right)= \\
& \left\{\begin{array}{l}
s_{2}\left(k, b_{m_{2}}, i\right)+\left(1-\frac{F_{2}}{3-2 F_{2}}\right)\left(r_{2}\left(k, b_{m_{2}}, i\right)\right. \\
\left.+q_{2}\left(k, b_{m_{2}}, i\right)\right), \text { if } m_{2}=1,2, \ldots 6 \\
s_{2}\left(k, b_{m_{2}}, i\right)+\left(1-F_{2}\right)\left(r_{2}\left(k, b_{m_{2}}, i\right)\right. \\
\left.+q_{2}\left(k, b_{m_{2}}, i\right)\right), \text { if } m_{2}=7,8, \ldots, 12
\end{array}\right. \\
& q_{2}\left(k, b_{m_{2}}, i+1\right)= \\
& \frac{F_{2}}{3}\left(r_{2}\left(k, b_{m_{2}+6}, i\right)+q_{2}\left(k, b_{m_{2}+6}, i\right)\right), \text { if } m_{2}=1,2, \ldots, 6 \\
& \frac{F_{2}}{3-2 F_{2}}\left(r_{2}\left(k, b_{m_{2}-6}, i\right)+q_{2}\left(k, b_{m_{2}-6}, i\right)\right) \\
& +\frac{F_{2}}{3}\left(r_{2}\left(k, b_{m_{2}+1}, i\right)+q_{2}\left(k, b_{m_{2}+1}, i\right)\right) \\
& +\frac{F_{2}}{3}\left(r_{2}\left(k, b_{m_{2}-1}, i\right)+q_{2}\left(k, b_{m_{2}-1}, i\right)\right) \text { if } m_{2}=8,9, \ldots, 11
\end{aligned}
$$$$
q_{2}\left(k, b_{m_{2}}, i+1\right)=
$$ 


$$
\left\{\begin{array}{l}
\frac{F_{2}}{3-2 F_{2}}\left(r_{2}\left(k, b_{m_{2}-6}, i\right)+q_{2}\left(k, b_{m_{2}-6}, i\right)\right) \\
+\frac{F_{2}}{3}\left(r_{2}\left(k, b_{m_{2}+1}, i\right)+q_{2}\left(k, b_{m_{2}+1}, i\right)\right) \\
+\frac{F_{2}}{3}\left(r_{2}\left(k, b_{12}, i\right)+q_{2}\left(k, b_{12}, i\right)\right), \text { if } m_{2}=7 \\
\frac{F_{2}}{3-2 F_{2}}\left(r_{2}\left(k, b_{m_{2}-6}, i\right)+q_{2}\left(k, b_{m_{2}-6}, i\right)\right) \\
+\frac{F_{2}}{3}\left(r_{2}\left(k, b_{7}, i\right)+q_{2}\left(k, b_{7}, i\right)\right) \\
+\frac{F_{2}}{3}\left(r_{2}\left(k, b_{m_{2}-1}, i\right)+q_{2}\left(k, b_{m_{2}-1}, i\right)\right), \text { if } m_{2}=12
\end{array}\right.
$$

The final propagation results of (1) and (2) can be obtained analytically by final value theorem. For example, if $M_{2}$ is 12 , the final propagation results at $b_{m_{2}}$ is

$$
\begin{aligned}
s_{2}\left(k, b_{m_{2}}, \infty\right)=\left[\begin{array}{llll}
P_{2,1, m_{2}} & P_{2,2, m_{2}} & \cdots & P_{2,12, m_{2}}
\end{array}\right] \\
\cdot\left[\begin{array}{llll}
r_{2}\left(k, b_{1}, 0\right) & r_{2}\left(k, b_{2}, 0\right) & \cdots & r_{2}\left(k, b_{12}, 0\right)
\end{array}\right]^{\mathrm{T}}
\end{aligned}
$$

where $P_{2,1, m_{2}}, P_{2,2, m_{2}}, \ldots$, and $P_{2,12, m_{2}}$ are the quantity of positions 1-12 affecting position $b_{m_{2}}$ within the two-dimensional pheromone basket and the values of $P_{2,1, m_{2}}$, $P_{2,2, m_{2}}, \ldots$, and $P_{2,12, m_{2}}$ are function of $F_{2}$.

\section{Two-Dimensional Pheromone Propagation CONTROLLER}

Relative to the food trail pheromones in the nature, the 2D-PPC employs "intercept pheromones" within the same wafer in the manufacturing process. The intercept pheromone assumes that the intercept of a measurement will affect others nearby within the same wafer at a run and the space-effect will maintain to affect the next wafer. So, the authors conduct 2D-PPC by interlacing space-effect controller and the traditional time-effect controller, such as PPC, in pairs.

\section{A. Plant}

The plant is the process model in a simulation or in a real system, which can be obtained by the linear regression model. This study assumes the process has 12 outputs and each output has its own disturbance and process gain. In addition, considering CMP process, pressure from inner and outer pad of wafer carrier are two inputs of the plant and measurements at the same circle has the same process gain. The demonstrated MIMO plant is

$$
Y_{k+1}=\alpha+\beta \cdot X_{k}+\varepsilon_{k+1} \quad k=0,1,2, \ldots
$$

where

$$
\begin{gathered}
Y_{k+\mathbf{1}}=\left[\begin{array}{llll}
Y_{1, k+1} & Y_{2, k+1} & \cdots & Y_{12, k+1}
\end{array}\right]^{T} \\
\alpha=\left[\begin{array}{llll}
\alpha_{1} & \alpha_{2} & \cdots & \alpha_{12}
\end{array}\right]^{T} \\
\beta=\left[\begin{array}{llll}
\beta_{1,1} & \beta_{2,1} & \cdots & \beta_{12,1} \\
\beta_{1,2} & \beta_{2,2} & \cdots & \beta_{12,2}
\end{array}\right]^{\mathrm{T}}
\end{gathered}
$$

$$
X_{k}=\left[\begin{array}{l}
X_{1, k} \\
X_{2, k}
\end{array}\right]
$$

and $\varepsilon_{k+\mathbf{1}}=\left[\begin{array}{llll}\varepsilon_{1, k+1} & \varepsilon_{2, k+1} & \cdots & \varepsilon_{12, k+1}\end{array}\right]^{T}$.

In addition, $Y_{k+1}$ are measurements at the end of run $k ; X_{k}$ are two recipes (inputs) of run $k ; \alpha$ are initial intercepts of the process; $\beta$ are system gains of run $k ; \varepsilon_{k+1}$ are disturbances, which include noise and uncontrolled terms of run $k+1$.

\section{B. The One-Dimensional Controller}

The one-dimensional controller is the traditional time effect controller, such as EWMA, double EWMA, PPC, and so on. Specifically, the one-dimensional controller of 2D-PPC control measurements independently in this study. For implementation, this study employs PPC [12] as the one-dimensional controller and the forecast intercept of measurement point $m_{2}$ at run $k+1$ becomes

$$
\begin{aligned}
& \tilde{\varepsilon}_{m_{2}, k+1}=2 \cdot v_{m_{2}, \mathrm{M}_{1}}(k)-v_{m_{2}, \mathrm{M}_{1}-1}(k) \\
& \quad=R_{1, m_{2}}^{T}(k, 0) \cdot P_{1, m_{2}, m_{2}}(k), \quad m_{2}=1,2, \ldots, M_{2}
\end{aligned}
$$

where

$$
\begin{aligned}
& R_{\mathbf{1}, \mathbf{m}_{2}}(k, i) \\
& \quad=\left[\begin{array}{llllll}
r_{1, m_{2}} & \left(k, b_{1}, i\right) & \cdots & r_{1, m_{2}}\left(k, b_{m_{1}}, i\right) & \cdots & r_{1, m_{2}}\left(k, b_{\mathrm{M}_{1}}, i\right)
\end{array}\right]^{T}
\end{aligned}
$$

is the external inputs of the measurements, $m_{2}$, at the pheromone basket of PPC with size $\mathrm{M}_{1}$ along time axis and

$$
\left\{\begin{array}{c}
r_{1, m_{2}}\left(k, b_{m_{1}}, 0\right)=\hat{\varepsilon}_{m_{2}, k-\left(\mathrm{M}_{1}-m_{1}\right)}-e_{m_{2}, k-\left(\mathrm{M}_{1}-m_{1}\right)} \\
\forall m_{1}=1, \ldots, M_{1}, \text { if } k>M_{1} \\
r_{1, m_{2}}\left(k, b_{m_{1}}, 0\right)=\hat{\varepsilon}_{m_{2}, m_{1}}-e_{m_{2}, m_{1}} \\
\forall m_{1}=1, \ldots, M_{1}, \text { if } k \leq M_{1}
\end{array}\right.
$$

where $\hat{\varepsilon}_{m_{2}, m_{1}}$ is forecast intercept of PPC at $m_{2}$ position and $m_{1}$ run; $e_{m_{2}, m_{1}}$ is the process error at $m_{2}$ position and $m_{1}$ run. In addition, $P_{1, m_{2}, m_{2}}(k)$, which varies with the size of the one-dimensional pheromone basket $\mathrm{M}_{1}$, means measurement $m_{2}$ affects $m_{2}$ itself by one-dimensional controller and is an algebraic expression of the propagation parameter of PPC at $m_{2}, F_{1, m_{2}}$.

\section{The Two-Dimensional Intercept Predictor}

The two-dimensional intercept predictor is the novel space effect controller, which modifies the forecasting intercepts obtained from the one-dimensional controller. The input of two-dimensional pheromone basket is the forecasting intercepts of the one-dimensional controller.

$$
\begin{aligned}
R_{2}(k, 0) & =\left[\begin{array}{llllll}
r_{2}\left(k, b_{1}, 0\right) & \cdots & r_{2}\left(k, b_{m_{2}}, 0\right) & \cdots & r_{2}\left(k, b_{\mathrm{M}_{2}}, 0\right)
\end{array}\right]^{T} \\
& =\left[\begin{array}{lllll}
\varepsilon_{1, k+1} & \cdots & \tilde{\varepsilon}_{m_{2}, k+1} & \cdots & \tilde{\varepsilon}_{M_{2}, k+1}
\end{array}\right]^{T}
\end{aligned}
$$

The forecast intercept at run $k+1, \hat{\boldsymbol{\varepsilon}}_{\mathbf{k}+\mathbf{1}}$, can be obtained by substituting (6) into (3). 


$$
\left[\begin{array}{c}
\hat{\varepsilon}_{1, k+1} \\
\hat{\varepsilon}_{2, k+1} \\
\vdots \\
\hat{\varepsilon}_{m_{2}, k+1} \\
\vdots \\
\hat{\varepsilon}_{M_{2}, k+1}
\end{array}\right]=\left[\begin{array}{cccccc}
P_{2,1,1} & P_{2,2,1} & \cdots & P_{2, m_{2}, 1} & \cdots & P_{2, M_{2}, 1} \\
P_{2,1,2} & P_{2,2,2} & \cdots & P_{2, m_{2}, 2} & \cdots & P_{2, M_{2}, 2} \\
\vdots & \vdots & \ddots & & & \vdots \\
P_{2,1, m_{2}} & P_{2,2, m_{2}} & & P_{2, m_{2}, m_{2}} & & P_{2, M_{2}, m_{2}} \\
\vdots & \vdots & & & \ddots & \vdots \\
P_{2,1, M_{2}} & P_{2,2, M_{2}} & \cdots & P_{2, m_{2}, M_{2}} & \cdots & P_{2, M_{2}, M_{2}}
\end{array}\right]\left[\begin{array}{c}
\tilde{\varepsilon}_{1, k+1} \\
\tilde{\varepsilon}_{2, k+1} \\
\vdots \\
\tilde{\varepsilon}_{m_{2}, k+1} \\
\vdots \\
\tilde{\varepsilon}_{M, k+1}
\end{array}\right]
$$

where the values of $P_{2,1,1}, P_{2,2,1}, \ldots$, and $P_{2, M_{2}, M_{2}}$ are function of $F_{2}$. If $F_{2}$ is 0 , the two-dimensional intercept predictor does not modify the forecasting intercept of the one-dimensional controller. In addition, the propagation result approaches the mean of the external inputs as $F_{2}$ approaches unity.

\section{Recipe Generator}

The recipe generator generates the recipe of the process for the next run. We use the linear regression model to produce

$$
T=\hat{\boldsymbol{\alpha}}+\hat{\boldsymbol{\beta}} \cdot X_{k+1}+\hat{\boldsymbol{\varepsilon}}_{k+1}
$$

where $X_{k+1} \in \mathfrak{R}$, which is a $2 \times 1$ matrix in this study, is the recipe (input) of run $k+1, \hat{\boldsymbol{\alpha}}$ is the estimator of the initial intercept $\alpha, \hat{\boldsymbol{\beta}}$ is the estimator of the system $\beta$ (model gain). Then, the recipe for run $k+1$ is

$$
X_{k+1}=\left(\hat{\boldsymbol{\beta}}^{T} \hat{\boldsymbol{\beta}}\right)^{-1} \hat{\boldsymbol{\beta}}^{T}\left(T-\hat{\boldsymbol{\alpha}}-\hat{\boldsymbol{\varepsilon}}_{k+1}\right)
$$

where $\hat{\boldsymbol{\alpha}}$ and $\hat{\boldsymbol{\beta}}$ are obtained from the linear regression model of the off-line DOE and are not equal to the process parameters $\alpha$ and $\beta$; the parameter $\hat{\boldsymbol{\varepsilon}}_{k+1}$ comes from (7), and $T$ is the given target values of measurements within a wafer.

\section{E. The Two-Dimensional Propagation Parameter Tuner}

This section proposes two strategies for tuning the two-dimensional propagation parameter $F_{2}$. The first strategy uses historical (or training) data and examines all possible $F_{2}$ values to obtain the best fixed propagation parameter, $\bar{F}_{2}$, with minimum mean square error:

$$
\bar{F}_{2} \cong \min _{F_{2}}\left(\sum_{k^{\prime}} \sum_{m_{2}} e_{m_{2}, k^{\prime}}^{2}\right)
$$

where $k^{\prime}$ is the index of the training data. Then, $\bar{F}_{2}$ is used in the testing data.

\section{Simulation Results}

The simulation settings included the following: the process model $\alpha$ and $\beta$ were

$$
\begin{aligned}
\alpha & =\left[\begin{array}{llllll}
\alpha_{1} & \cdots & \alpha_{6} & \alpha_{7} & \cdots & \alpha_{12}
\end{array}\right]^{T} \\
& =\left[\begin{array}{llllll}
6700.26 & \cdots & 6700.26 & 4304.63 & \cdots & 4304.63
\end{array}\right]^{T}
\end{aligned}
$$

and

$$
\begin{aligned}
\beta & =\left[\begin{array}{llllll}
\beta_{1,1} & \cdots & \beta_{6,1} & \beta_{7,1} & \cdots & \beta_{12,1} \\
\beta_{1,2} & \cdots & \beta_{6,2} & \beta_{7,2} & \cdots & \beta_{12,2}
\end{array}\right]^{T} \\
& =\left[\begin{array}{cccccc}
-1356.63 & \cdots & -1356.63 & 85.54 & \cdots & 85.54 \\
1182.19 & \cdots & 1182.19 & -1458.8 & \cdots & -1458.8
\end{array}\right]^{T}
\end{aligned}
$$

the initial input was $[1,0]$, the process target $T$ was $4500 \mathrm{~nm}$, and the offset of controller model $(\hat{\boldsymbol{\alpha}}, \hat{\boldsymbol{\beta}})$ will be specified in the beginning of following section respectively. Two performance indices to evaluate the output performance: average (Ave.) and standard deviation (Std.) of the simulated data.

\section{A. Performance Comparison with and Without 2D-PPC}

This section compares output performance of nine candidate controllers with model mismatch $\xi=1.1$. The fixed optimal $1 \mathrm{D} / 2 \mathrm{D}$ propagation parameter or weights of the other controllers were obtained using the minimum mean square error from 27 sets of anthropogenic disturbance and one set of anthropogenic disturbance is shown in Fig. 2. The simulation results are listed at Table I. In Table I, the output performance of PPC with 2D-PPC is better in both average Ave. and Std. within a wafer than the one of using PPC alone.

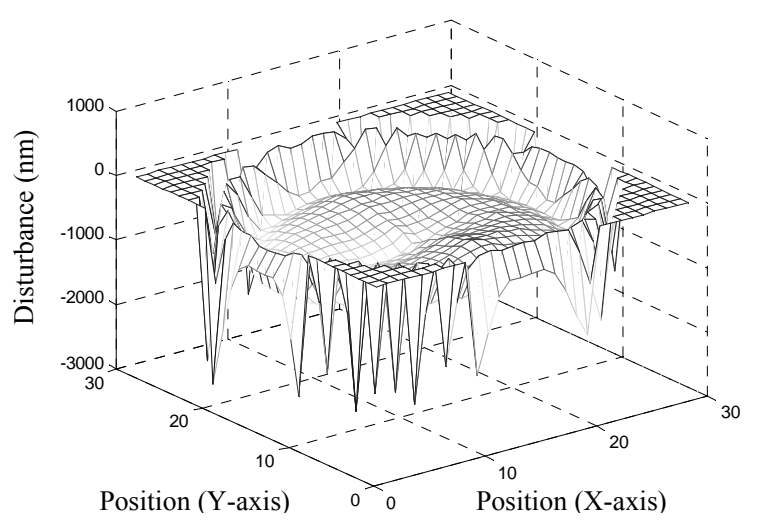

Fig. 2. One set of anthropogenic disturbance.

TABLE I: SimULATION RESULTS With $\xi=1.1$.

\begin{tabular}{ccc}
\hline \hline & PPC & PPC with 2D-PPC \\
\hline Average Ave. within a wafer (nm) & 4368.67 & 246.21 \\
Average Std. within a wafer (nm) & 4435.31 & 220.91 \\
Improvement (\%) & 50.7 & 10.3 \\
\hline \hline
\end{tabular}

\section{CONCLUSION}

The 2D-PPC modifies forecasting intercept of the linear regression model of traditional time-effect (or one-dimensional) controller. Dealing with high order disturbances and propagating "healthy" intercepts to neighbors are the advantage of 2D-PPC. Simulation results shows that one of the 2D-PPC can improve the proposed one-dimensional controller both in average Ave. and Std. within a wafer when $\xi=1.1$. 


\section{ACKNOWLEDGMENT}

The authors would like to thank the National Science Council of the Republic of China for financially supporting this paper under Contract NSC 100-2221-E-009-063-MY2.

\section{REFERENCES}

[1] E. Sachs, A. Hu, and A. Ingolfsson, "Run by run control: Combining SPC and feedback control," IEEE Trans. Semicond. Manuf., vol. 8, pp. 26-43, Feb. 1995.

[2] J. A. Stefani, S. Poarch, S. Saxena, and P. Mozumder, "Advanced process control of a CVD tungsten reactor," IEEE Trans. Semicond. Manuf., vol. 9, no. 3, pp. 366-383, 1996.

[3] J. Wang, Q. P. He, S. J. Qin, C. A. Bode, and M. A. Purdy, "Recursive Least Square Estimation for Run-to-Run Control with Metrology Delay and Its Application to STI Etch Process," IEEE Trans. Semicond. Manuf., vol. 18, pp. 309-319, May 2005.

[4] A. Chen and R. S. Guo, "Age-based double EWMA controller and its application to CMP processes," IEEE Trans. Semicond. Manuf., vol. 14, no. 1, pp. 11-19, Feb. 2001.

[5] N. S. Patel and S. T. Jenkins, "Adaptive optimization of run-to-run controllers: The EWMA example," IEEE Trans. Semicond. Manuf., vol. 13, pp. 97-107, 2000.

[6] E. D. Castillo, "Long run and transient analysis of a double EWMA feedback controller,” IIE Trans., vol. 31, no. 12, pp. 1157-1169, 1999.

[7] A. C. Lee, Y. R. Pan, and M. T. Hsieh, "Output Disturbance Observer Structure Applied to Run-to-Run Control for Semiconductor manufacturing," IEEE Trans. Semicond. Manuf., vol. 24, no. 1, pp. 27-43, Feb. 2011.

[8] J. H. Chen, T. W. Kuo, and A. C. Lee, "Run-by-run process control of metal sputter deposition: combining time series and extended Kalman filter," IEEE Trans. Semicond. Manuf., vol. 20, no. 3, pp. 278-285, 2007.

[9] C. F. Wu, C. M. Hung, J. H. Chen, and A. C. Lee, "Advanced Process Control of the Critical Dimension in Photolithography," Int. J. Precis. Eng. Manuf., vol. 9, no. 1, pp. 12-18, 2008.

[10] T. W. Kuo and A. C. Lee, "Assessing Measurement Noise effect in Run-to-Run Process Control: Extends EWMA Controller by Kalman Filter," Int. J. Automation and Smart Technology, vol. 1, no. 1, pp. 67-76, Sep. 2011.
[11] S. Brueckner, "Return from the Ant: Synthetic Ecosystems for Manufacturing Control," Ph.D. dissertation, Humboldt University of Berlin, 2000.

[12] D. S. Lee and A. C. Lee, "Pheromone Propagation Controller: the Linkage of Swarm Intelligence and Advanced Process Control," IEEE Trans. Semicond. Manuf., vol. 22, no. 3, pp. 357-372, Aug. 2009.

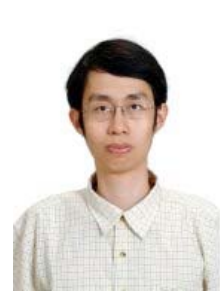

Der-Shui Lee received the B.S. degree in mechanical engineering from the Nation Chung-Hsing University, Tai-Chung, Taiwan, in 1997 and the M.S. degree in mechanical engineering from the National Chiao-Tung University, HsinChu, Taiwan, in 1999 and the Ph.D. degree in 2012 from National Chiao-Tung University in mechanical engineering, HsinChu, Taiwan. After graduation, since 2000, he served as an Assistant Researcher in Chung-shan institute of Science and Technology (CSIST). His research interests include run-to-run (R2R) process control, swarm intelligence, and decision support system.

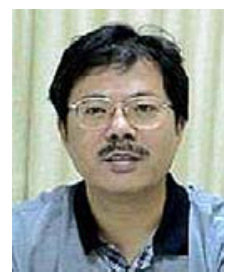

An-Chen Lee received the B.S. and M.S. degrees in Power Mechanical Engineering from National Tsing-Hua University, Hsinchu, Taiwan, R.O.C. and the $\mathrm{Ph} . \mathrm{D}$. degree in 1986 from University of Wisconsin-Madison in Mechanical Engineering, USA. He is designated as Chair Professor of National Chiao-Tung University and currently a Professor in the department of Mechanical Engineering. His current research interests are $\mathrm{CNC}$ machine tool control technology, Magnetic bearing technology, Rotor dynamic and control, and Semiconductor manufacturing process control.

Prof. Lee served as an Editorial Board member of international Journal of Precision Engineering and Manufacturing, Chinese society of Mechanical Engineers. He is the recipient of National Science Committee (NSC) Excellent Research Award (1991-1992), NSC Distinguished Research Award (1993-1994, 1995-1996, 1997-1998), NSC research fellow (1999-2001, 2002-2004), NSC research fellow Award (2005), Chinese Society of Mechanical Engineers Distinguished Engineering Professor Award (2001), Gold Medal Award, Inventor/New Product Exposition (INPEX), Pittsburgh, U.S.A. (2010), Gold Medal Award, International Exhibition of Inventions of Geneva (2011). 